\title{
Sostenibilidad en agroecosistemas de café de pequeños agricultores: revisión sistemática
}

\author{
Sustainability in coffee agroecosystems of small farmers: a systematic review \\ Mónica María Machado Vargas ${ }^{1}$, Leonardo Alberto Ríos Osorio ${ }^{2 *}$
}

\begin{abstract}
RESUMEN
El propósito de este artículo fue realizar una revisión sistemática respecto de indicadores para estudiar y evaluar agroecosistemas de café en pequeños agricultores. Dentro de la literatura encontrada se observó dos marcos referenciales: las áreas técnico-ambientales de los cuales se derivan indicadores como prácticas de protección del medio ambiente y producción; y las áreas sociales con indicadores para evaluar los medios de vida, bienestar y lo referente a capital natural, capital humano, capital social, capital físico y capital financiero. De acuerdo con esto, emergen indicadores para analizar la capacidad adaptativa de las poblaciones rurales para ser resilientes a los cambios sociales, ecológicos y económicos. Estos indicadores deben relacionar lo social, lo económico y el sistema ecológico, e interaccionar en una escala temporal, espacial y organizacional.
\end{abstract}

Palabras claves: sostenibilidad, indicadores, café, pequeños agricultores.

\begin{abstract}
The purpose of this paper was to conduct a systematic review of indicators to study and evaluate agro coffee smallholders. Within the found literature, two frames of reference were observed: the technical-environmental areas in which indicators and practical protection of the environment and production are derived; and social areas with indicators to assess livelihoods, welfare and all related to natural capital, human capital, social capital, physical capital and financial capital. Accordingly, indicators emerge to analyze the adaptive capacity of rural communities to be resilient to the social, ecological and economic changes. These indicators have to include social, economic and ecological dimensions, and interact in a temporal, spatial, and organizational scale.

Key words: sustainability, indicators, coffee, small farmers.
\end{abstract}

\section{Introducción}

En los últimos años, la creciente conciencia acerca del impacto ambiental, social y cultural de prácticas de la agricultura moderna como es el uso ineficiente de insumos sintéticos derivados del petróleo, ha ocasionado erosión y salinización de suelos, contaminación de aguas y aumento de gases de efecto invernadero; otras prácticas han ocasionado la pérdida de cultivos tradicionales, aumento de plagas y enfermedades, entre otros (Altieri, 2008). Asimismo, el aumento del área para la agricultura ha ocasionado pérdida de hábitats y su biodiversidad asociada, y con ellos la pérdida de los servicios ecosistémicos (Pretty, 2008). También la agricultura moderna ha aumentado la marginación de la población rural que ve deteriorarse sus niveles de vida, particularmente en lo que respecta a las condiciones de trabajo y a los niveles de alimentación (Altieri y Toledo, 2011).

No está ajeno a esto la caficultura en el mundo. A mediados del siglo pasado las plantaciones de café que se establecieron tenían la estrategia denominada "cafetal bajo monte raleado", es decir, se aprovechaba la sombra de las especies arbóreas nativas del bosque primario para la producción de café. A partir de los años sesenta, la adopción de nuevas tecnologías con la llegada de la Revolución Verde, orientadas a intensificar la caficultura por medio del uso intensivo de agroquímicos y la eliminación del sombrío del cafetal, transformó los cafetales diversificados en monocultivos (Fischersworring y Robkamp, 2001).

1 Grupo de investigación Medio Ambiente y Sociedad, Universidad de Antioquia. Sede de Investigación Universitaria, torre 1, oficina 213, tel. 574 2196479. Medellín, Colombia.

2 Universidad de Antioquia, Grupo de Investigación Salud y Sostenibilidad. Medellín, Colombia.

* Autor para correspondencia: leonardo.rios@udea.edu.co

Fecha de Recepción: 10 Octubre, 2015.

Fecha de Aceptación: 26 Noviembre, 2015. 
En los últimos años se ha hecho énfasis en el deterioro de la biodiversidad en la conversión del café de sombra al café de sol. Estudios han demostrado que los elementos de biodiversidad, incluyendo las plantas, reptiles, aves, insectos y algunos mamíferos, son más altos en el café con sombra que en el café sin sombra (Borkhataria et al., 2012). La conservación de la biodiversidad como son las hormigas, aves y lagartos pueden disminuir la abundancia de insectos plagas en el café (Perfecto et al, 2004; Borkhataria et al., 2012). Los árboles como sombrío en el cultivo de café constituye una práctica agroecológica que proporciona servicios ecosistémicos como, refugio y hábitat para la biodiversidad, secuestro de carbono, regulación del microclima y fijación de nitrógeno, además de ser otra fuente de ingreso para los caficultores (Meylan et al., 2013).

Un estudio realizado en Centroamérica acerca de las estrategias utilizadas por los pequeños productores de café que les permitió un cierto nivel de éxito para superar las fluctuaciones de los precios internacionales encontró que entre las más efectivas implementadas estaba la producción y comercialización de cafés especiales y la diversificación de la producción de café por la introducción de rubros complementarios; esto implicó la aplicación de prácticas agroecológicas para mejorar la calidad y asegurar la naturaleza orgánica del producto (Damiani, 2005). Actualmente el café es considerado uno de los principales commodities en el mundo, con plantaciones de café que cubren aproximadamente 10,6 millones de hectáreas de tierra, casi toda en los trópicos y la producción soporta el sustento de vida de cuatro millones de pequeños productores en 14 países (Castro-Tanzi, 2012).

Por todo lo anterior, se ha llegado a plantear la necesidad de un cambio en la producción de alimentos, es decir, una agricultura sostenible. De ahí la necesidad de estudiar la complejidad de los problemas de insostenibilidad de los sistemas agrarios desde un nuevo enfoque sistémico. Este enfoque holístico, que permite entender la problemática agrícola en términos sistémicos se denomina agroecología, esta estudia las relaciones entre los sistemas agrícolas y el medio ambiente y la sociedad dentro de las que se desarrollan tales sistemas (Altieri, 1999). Por tanto, la agroecología estudia las relaciones socioecológicas de los agroecosistemas, entendiendo los sistemas socioecológicos como sistemas constituidos por los subsistemas sociales, económicos, institucionales y ecológicos, y las relaciones entre ellos (Gallopín, 2003). Es por esto que la agricultura sostenible no debe soportarse solamente en la ecología del agroecosistema específico donde se practica, sino que debe trascender las estructuras culturales y sociales en las que el sistema se desarrolla (HoltGiménez, 2008).

Actualmente, uno de los desafíos que enfrentan tanto agricultores como extensionistas e investigadores es saber en qué estado se encuentra el agroecosistema. Por esto, algunos especialistas (Hart, 1985; Masera et al., 1999; Astier et al., 2008; Sarandón, 2002) han diseñado metodologías para la evaluación de agroecosistemas que permite monitorear la evolución de un mismo agroecosistema en el tiempo, para que tanto el agricultor como el investigador o extensionista puedan determinar el estado del mismo y con ello realizar una planeación y un diseño sostenible. En este sentido, Altieri y Nicholls (2002) desarrollaron una metodología rápida para evaluar el agroecosistema de café de pequeños productores en Costa Rica, los indicadores para la evaluación se construyeron de forma participativa y de acuerdo con las necesidades y conocimientos de los pequeños caficultores. Es importante a la hora de hacer una evaluación de sostenibilidad de un agroecosistema de café, en seleccionar unos indicadores que reflejen la integración social, ambiental y económica, así como sus interrrelaciones (Sarandón y Flores, 2009), de esta forma permite tener una visión holística del agroecosistema.

Es por esto, que el objetivo de este artículo fue describir los indicadores para el estudio y evaluación de la sostenibilidad de pequeños productores de café a partir de una revisión sistemática de la literatura científica.

\section{Materiales y Métodos}

\section{Estrategia de búsqueda}

El estudio se llevó a cabo en conformidad con la declaración PRISMA (Urrútia y Bonfill et al., 2010). En julio de 2014 se realizó una búsqueda sistemática de literatura en las bases de datos ScienceDirect, Springer Link y Scopus, por sensibilidad, utilizando descriptores DeCS, por exhaustividad, utilizando descriptores no DeCS y por especificidad, utilizando la combinación mediante operadores booleanos de 
términos definidos de acuerdo con la pregunta de investigación.

La búsqueda de la ruta general utilizada fue: [((coffee smallholder) AND indicator)) AND (agroecology OR sustainability)]. En las bases de datos ScienceDirect, Springer Link y Scopus se utilizaron los límites de tiempo "2004 to present" y "between 2004 and 2014".

Las citas encontradas junto con su respectivo resumen se importaron al software de gestión de referencias EndNote, en el que se eliminaron las citas duplicadas entre las bases de datos. El protocolo de búsqueda fue aplicado por dos revisores de forma independiente, cuyas diferencias fueron analizadas $\mathrm{y}$ resueltas por mutuo acuerdo.

\section{Criterios de inclusión y exclusión}

Se incluyeron solo artículos originales reportados en la literatura científica en los últimos 10 años (2004 a 2014), escritos en el idioma inglés. Los artículos fueron estudios relativos a la evaluación de sistemas de producción de café de pequeños productores que utilizaron indicadores de sostenibilidad o agroecológicos. Además se tuvieron en cuenta artículos que describieran la categoría de análisis del sistema de producción y tipo de productor; describiera el indicador y su nivel de medición y describiera el marco referencial del concepto de sostenibilidad. Se excluyeron los artículos que solo contemplaran los grandes productores de café. Los datos de cada publicación fueron extraídos y tabulados para su posterior análisis, en una base de datos en Excel.

\section{Resultados y Discusión}

La implementación del protocolo de búsqueda mediante las rutas descritas previamente arrojó 218 artículos publicados entre 2004 y 2014 (ScienceDirect 52, Springer Link 67 y Scopus 99). Posteriormente se eliminaron nueve referencias duplicadas entre las bases de datos (gestor de referencias EndNote), se evaluaron 209 publicaciones con base en el título y el resumen, de estas 190 fueron descartadas por no cumplir con los criterios de inclusión determinados de acuerdo con la pregunta de investigación. Se analizaron 19 artículos en texto completo y como resultado tres fueron eliminados por cumplir con los criterios de exclusión. Basado en lo anterior, el protocolo de búsqueda en las tres bases de datos incluyó 15 artículos en la revisión sistemática (Figura 1).

A estos 15 artículos obtenidos se le sumó literatura gris que fue obtenida mediante la herramienta de búsqueda Google Académico, con 12 tesis de doctorado para garantizar la exhaustividad, de estas solo tres cumplen con los criterios de inclusión y nueve se descartaron por cumplir con los criterios de exclusión. Los artículos seleccionados son investigaciones de África, Asia, Centro y Sur América (Figura 2).

\section{Sistema de producción de los pequeños agricultores de café}

De acuerdo con la literatura encontrada, autores como Katlyn et al., 2013; Bacon et al., 2014 afirman que la mayoría de los productores de café en el mundo son pequeños agricultores, en este sentido, el $85 \%$ de los caficultores en Centroamérica son pequeños y en su mayoría indígenas. Estos pequeños caficultores se caracterizan por poseer áreas mínimas de producción, como se evidencia en América Latina, países como Nicaragua donde la gran mayoría son pequeños productores con 3,5 ha que producen el $85 \%$ de la cosecha de café (Donovan y Poole, 2014). En Costa Rica las áreas mínimas son 0,17 ha y máximo 4,9 ha (Castro-Tanzi, 2012), mientras que en Perú los pequeños caficultores poseen de 5 a 6 hectáreas y generalmente utilizan cerca de 0,5 hectáreas para cultivos, huertas familiares y ganadería a pequeña escala o estabulada (Ruben y Fort, 2012). En África, en países como Rwanda, el tamaño promedio es de 0,76 ha y en monte Kilimanjaro, Tanzania, el tamaño promedio de esta zona es de 0,56 ha, pero la mitad de los agricultores tienen menos de 0,4 ha, los que son considerados chacras o huertos caseros (Soini, 2005; Elder et al., 2013).

Según Bacon et al. (2014), una de las características importantes de los pequeños caficultores son los agroecosistemas diversificados o sistemas agroforestales, los que proporcionan servicios ecosistémicos. Este mismo autor sugiere que las primeras tierras con café bajo sombra se dieron en El Salvador, Guatemala, Nicaragua y parte de México (Bacon et al., 2012). En la literatura encontrada autores como Bacon et al., 2012; Katly et al., 2013; Speelman et al., 2014, sugieren que estos sistemas agroforestales de café se caracterizan por la conservación de la biodiversidad y conservación de remanentes de bosques; los árboles le proporcionan 


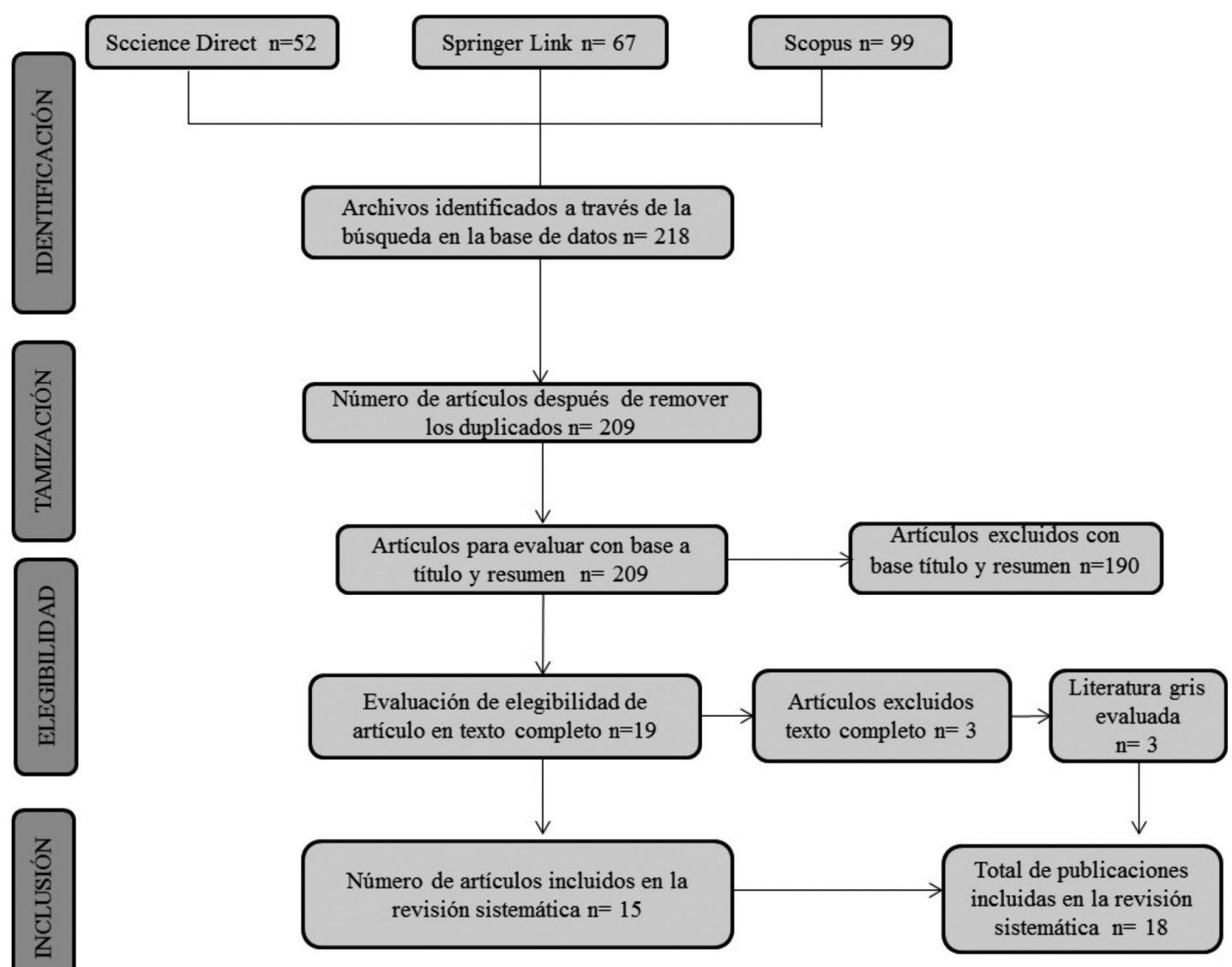

Figura 1. Flujograma estrategia de búsqueda (Urrútia y Bonfill et al., 2010).

al agricultor una variedad de productos como frutas, leña y madera. Los pequeños caficultores también tienen parcelas de subsistencia de maíz y frijol que son parte de su dieta, otras parcelas contienen pequeñas cantidades de variedades de vegetales para consumo o ventas ocasionales y plantas medicinales; también se resalta en estos sistemas agroforestales la calidad del café (Bacon et al., 2012; Katly et al., 2013; Speelman et al., 2014).

En Tanzania, monte Kilimanjaro, Soini (2005), describen los sistemas agroforestales tradicionales de café en sistemas combinados de café-banano, con múltiples otros cultivos de comida, cultivos intercalados y cultivos de maíz, mijo y frijol. Los árboles son utilizados para protección del café, como cercas vivas, para forraje y mulch, para alimento de abejas, para madera y leña. También las colmenas y gallinas juegan un papel muy importante dentro de estos sistemas. Por otro lado, los sistemas de café a pequeña escala en Rwanda y Brasil descritos por Nonato de Souza et al., 2012; Elder et al., 2013, respectivamente, estos sistemas de café en su mayoría crecen sin sombra, el que requiere de altas cantidades de pesticidas y fertilizantes químicos.

Según Moguel y Toledo, 1999, en un estudio realizado en México, los sistemas de producción de café se clasifican de acuerdo con su vegetación y complejidad de la estructura de sus policultivos tradicionales o comerciales. En los policultivos complejos tradicionales el café crece junto a especies de plantas nativas o introducidas que incluye remanentes de bosque y en doseles de 20-30 m de altura. Los sistemas policultivos no tradicionales se caracterizan por una completa remoción de los árboles de los bosques originales y la introducción de especies de árboles para sombra que son apropiados para los cultivos de café y que frecuentemente son plantas fijadoras de nitrógeno como Inga sp. y con doseles menos de 15 metros (Rahn et al., 2013). 


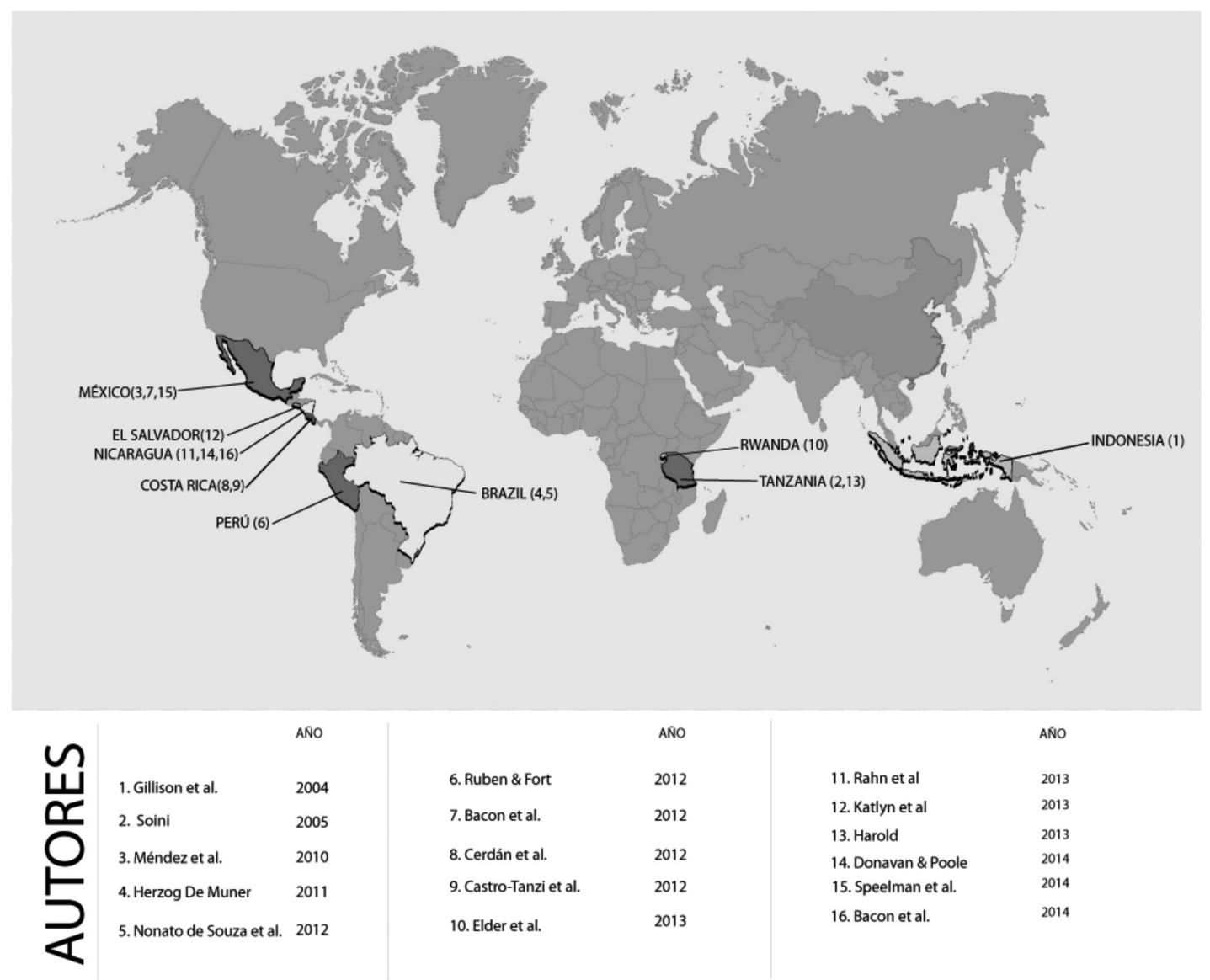

Figura 2. Mapa con las áreas de estudio, sus autores y año de publicación, encontrada en la revisión sistemática.

\section{Concepto de sostenibilidad para evaluar agroecosistemas de café}

En los estudios seleccionados se encontró que la descripción del concepto de sostenibilidad se divide en dos marcos referenciales, una desde las áreas técnicas ambientales y la otra en las áreas de lo social.

Según Castro-Tanzi (2012), consideró que los agroecosistemas de café más sostenibles son aquellos que presentan características con mayor eficiencia en el uso de fertilizantes orgánicos, ligado a esto la calidad del suelo y por tanto el rendimiento del café y la calidad de taza de café, con lo anterior, los caficultores pueden reducir los costos de producción y mejorar sus estilos de vida mientras mantengan o mejoren la producción y calidad.

Otros autores (Gillison et al., 2004; Nonato de Souza et al., 2012; Cerdán et al., 2012; Bacon et al.,
2012) se refieren a los sistemas con agrobiodiversidad como es el caso de los sistemas agroforestales de café, que contribuyen a la conservación de la biodiversidad y los servicios ecosistémicos, como estabilidad del clima, suplemento del agua, regulación de las inundaciones, polinización de los cultivos, fertilidad y conservación del suelo, control de enfermedades y plagas, secuestro de carbono, oportunidades de recreación, esparcimiento y cultura, como sistemas que mejoran la sostenibilidad y resiliencia de los sistemas de agricultura.

Para el autor Cerdán et al. (2012) los servicios ecosistémicos y la conservación de la biodiversidad en los sistemas agroforestales de café frecuentemente se han estudiado por separado desde su productividad, entradas y salidas, la rentabilidad, el precio del café y los salarios locales. Bacon et al. (2012) resalta la importancia de introducir las dimensiones sociales para el estudio de los agroecosistemas de café, ya 
que existe una interdependencia entre los ecosistemas y el sistema socioeconómico-cultural en lo local, regional, nacional, continental y global, a esto se suma el desarrollo de un marco referencial que usa la interdisciplinariedad para evaluar los sistemas sociecológicos. Por tanto, identificar las variables sociales para el estudio complejo de las realidades socioambientales de los agroecosistemas de café, requiere una multidisciplinariedad (Bacon et al., 2012).

Otro autor como Katlyn et al., (2013) propone estudiar la sostenibilidad de los medios de vida rural analizada en lo referente a la pobreza, inseguridad alimentaria y la ausencia de acceso a recursos, siendo la inseguridad alimentaria un indicador de inestabilidad e insostenibilidad de los medios de vida. Los medios de vida son la capacidad de adquirir varias actividades de subsistencia y se nombran como capital humano, capital natural, físico, social y financiero, que podrían ser individuales o colectivos (Donovan y Poole, 2014). Además de permitir a los agricultores tener estrategias para ganar mejores estilos de vida, como más ingresos, incremento del bienestar, reducción de la vulnerabilidad, entre otros (Soini, 2005; Mendén et al., 2010). Según Katlyn et al. (2013), para evaluar los medios de vida se debe tener en cuenta lo social, cultural y el contexto político, enfatizando en las políticas globales y locales, la globalización, las redes de mercado y los desafíos ambientales.

El término de sostenibilidad de los medios de vida generalmente se refiere a la resiliencia de los agricultores ante el estrés o perturbaciones ambientales, económicas y sociales (Katlyn et al., 2013). La diversificación de los medios de vida está relacionado con las estrategias de cómo las poblaciones rurales pueden manejar el riesgo, mantener la resiliencia, enfrentar y adaptarse al estrés o las perturbaciones (Katlyn et al., 2013). Mejorar la capacidad adaptativa de los agricultores y las comunidades es esencial para el futuro sostenible de los medios de vida rurales, siendo definida la capacidad adaptativa como la habilidad individual o colectiva para modificar el manejo sostenible de los recursos naturales en respuesta a lo actual, mejorar la resiliencia de un sistema y reducir su vulnerabilidad, esto permite pasar de un sistema en estado indeseado o un sistema deseado (Speelman et al., 2014). La capacidad adaptativa de un sistema es fundamental para desarrollar estrategias adaptativas que frecuentemente están basadas en redes sociales, reorientación en la producción agrícola, mejorar la infraestructura, mejorar la estructura de organización local y diversificar la producción del sistema. Sin embargo, estas estrategias dependen de la disponibilidad de recursos, que incluye respuestas económica e institucional que afectan los sistemas socioeconómicos (Speelman et al., 2014).

Según Danse y Vellema (2007), este creciente interés por incorporar el concepto de sostenibilidad en el comercio internacional de la producción de café se debe en parte por la presión que los compradores internacionales de café, principalmente los tostadores, ejercieron sobre los proveedores para cumplir con los nuevos requerimientos de los consumidores respecto de las condiciones ambientales y sociales en los años 90 con estándares que combinan la calidad del café y los procesos de producción sostenibles. Para Elder et al. (2013), el marco de la producción cafetera está medida por tres ejes fundamentales que contemplan lo social, ambiental y lo económico y cada una de las certificadoras enfatizan bien que sea en uno de estos aspectos como mínimo o en todos, siendo el caso ideal. De ahí la creación de nuevos nichos de mercado que tienen estándares ambientales específicos para la protección del medio ambiente, salud laboral y seguridad (Elder et al., 2013) y otros para la preservación de bosques y aves.

Por tanto, autores como Danse y Vellema, 2007; Méndez et al., 2010; Ruben y Fort, 2012; Elder et al., 2013; Katlyn et al., 2013; Donovan y Poole, 2014; Bacon et al., 2014; han realizado estudios para entender cómo las certificadoras internacionales de café están influenciando o afectando los medios de vida de los pequeños productores, su cultura y las condiciones ambientales, es por esto que estos autores han desarrollado varios indicadores para este propósito.

\section{Indicadores para evaluar agroecosistemas de café}

Basados en el marco referencial para estudiar la sostenibilidad de los agroecosistemas de café, en esta revisión sistemática se encontró que algunos autores proponen indicadores técnico-ambientales para conocer el estado actual de un agroecosistema, mientras que otros autores combinan indicadores técnico-ambientales y socioeconómicos.

Los indicadores técnico-ambientales se refieren a prácticas de protección del medio ambiente y producción, autores como Gillison et al., 2004; Soini, 2005; Nonato de Souza et al., 2012; Cerdán et al., 
2012; Elder et al., 2013; Rahn et al., 2013 sugieren atributos como composición florística, microclima, calidad de suelo, calidad de hojarasca, uso de fertilizantes, uso de pesticidas, número de cultivos, rendimiento del café, ingresos de la finca, manejo del dosel de los árboles de sombra, clasificación de los árboles en la finca, clasificación de mamíferos y aves en la finca, prácticas de conservación de agua y suelo, uso de coberturas, productividad y calidad del café, prácticas para mitigar el cambio climático, entre otros.

Además, los indicadores socioeconómicos más utilizados por los autores de esta revisión (Soini, 2005; Ruben y Fort, 2012; Bacon et al., 2012; Katlyn et al., 2013; Rahn et al., 2013; Donovan y Poole, 2014; Hauserman, 2014; Speelman et al., 2014) fueron basados en atributos para evaluar los medios de vida, bienestar y lo referente a capital natural, capital humano, capital social, capital físico y capital financiero. Algunos atributos que se pueden resaltar de la literatura revisada fueron: grado de inseguridad alimentaria, migración, ingresos del hogar, educación, redes sociales, vulnerabilidad al cambio climático, instituciones sociales y organizaciones, demografía del hogar, uso de la tierra, salud y nutrición, entre otros.

Según Katlyn et al. (2013), los caficultores invierten más tiempo y energía en la producción de café que en producir alimentos para su seguridad alimentaria, ello da como resultado que muchos pequeños caficultores y sus familias pasen periodos de hambre, a esto se suma la pobreza, la ausencia de educación, la falta de infraestructura y de redes de mercado. Es bien sabido que la vulnerabilidad de los pequeños caficultores se debe en parte a los altos costos de la producción de café y la fluctuación de los precios internacionales, y en algunos casos a la tenencia de la tierra o cambios en el uso de la tierra (Hausermann, 2014).

Por lo anterior, es importante examinar esos cambios económicos, institucionales, en el uso de la tierra y la respuesta de las comunidades a la reorganización social, asimismo estudiar las estrategias y diversificación de los medios de vida, la capacidad adaptativa y la resiliencia. Actualmente la capacidad adaptativa es comúnmente analizada y discutida en el contexto del cambio climático, sin embargo, el concepto puede ser utilizado más ampliamente, ya que incluye una respuesta económica, institucional, que afecta los sistemas socioecológicos (Speelman et al., 2014). Por tanto, los indicadores para analizar la sostenibilidad de los agroecosistemas de café se deben relacionar con lo social, económico y el sistema ecológico, que interacciona en una escala temporal, espacial y organizacional. Es por esto que la sostenibilidad de la agricultura no es fácil de delimitar en una parcela, campo, finca o en una escala de tierra porque está inmersa dentro de un gran sistema socioecológico (Bacon et al., 2012).

Por consiguiente, si partimos de un marco referencial como la sostenibilidad de los medios de vida, han surgido algunos atributos emergentes para evaluar las estrategias de adaptación de las comunidades y su resiliencia, como: Redes sociales, reorientación de producción agrícola, infraestructura, organización local, diversificación de los medios de vida, diversificación de los sistemas de producción, disponibilidad de recursos, instituciones y organizaciones, vulnerabilidad al cambio climático, seguridad alimentaria, salud y nutrición, educación, redes de mercado, tenencia de la tierra y contexto político (políticas globales y locales). Se espera que estos atributos puedan tener un acercamiento a la realidad compleja que viven los pequeños caficultores en el mundo, estos están bajo la presión de los cambios en los precios del mercado internacional, las presiones políticas, de uso de la tierra y tenencia de la tierra, así como a los cambios climáticos.

\section{Conclusiones}

En la literatura científica se encuentran dos modelos de evaluación de la sostenibilidad de sistemas de pequeños productores de café: uno que se basa en un concepto de sostenibilidad relacionado con los beneficios de los servicios ecosistémicos y las prácticas ambientales asociados a la calidad del café, abordaje más relacionado con una visión ecologista de la sostenibilidad. El otro modelo se basa en una concepción de sostenibilidad más relacionada con los medios o modos de vida de los pequeños productores, generando una línea base para valorar la capacidad adaptativa de las poblaciones rurales frente a los cambios sociales, ecológicos y económicos del entorno.

Otra de las características encontradas en la literatura es la carencia de información tanto de las variables involucradas en la concepción de sostenibilidad a evaluar como de la serie de tiempo de los datos necesarios para conocer mejor esta condición, ello evidencia que se hace necesario 
generar sistemas de información acordes con las concepciones de sostenibilidad que se están desarrollando en el sector agrícola.

Igualmente, la envergadura de los sistemas productivos está directamente relacionada con las propuestas de indicadores de sostenibilidad, observándose cómo para sistemas de pequeños productores se encuentran tendencias en los indicadores más relacionadas con aspectos socioculturales de la producción agrícola, asociados a la concepción de agricultura familiar, que integra la productividad con los estilos de vida de los productores.
De acuerdo con lo anterior, se hace necesario desarrollar sistemas de indicadores que trasciendan atributos independientes de los sistemas productivos y se acerquen a propuestas de índices o marcos de evaluación, que deriven de conceptos de sostenibilidad integradores de los aspectos productivos y los aspectos socioculturales, concertados con las comunidades objeto de evaluación.

Finalmente, las revisiones sistemáticas son una herramienta útil para conocer el panorama actual de un área específica de conocimiento, como base para poder desarrollar preguntas de investigación más pertinentes para el avance de la ciencia.

\section{Literatura Citada}

Altieri, M.A.

1999. Agroecología, Bases Científicas para una Agricultura Sustentable. Montevideo: Nordan-Comunidad. pp. 325.

Altieri, M.A.; Nicholls, C.I.

2002. Un método agroecológico rápido para la evaluación de sostenibilidad en cafetales. Manejo Integrado de Plagas y Agroecología, 64: 19-24.

Altieri, M. y Nicholls, C.

2008. Optimizando el manejo agroecológico de plagas a través de la salud del suelo. Revista Agroecología, ISSN electrónico: 1989-4686, 1: 29-36.

Altieri M.A. y Toledo,V.

2011. The agroecological revolution in Latin America: rescuing nature, ensuring food sovereignty and empowering peasants. The Journal of Peasant Studies, 38 (3): 587-612.

Astier, M.; Masera, R.O.; Galván-Miyoshi, Y.

2008. Evaluación de sustentabilidad. Un enfoque dinámico y multidimensional. Seae, Ciga, Ecosur, Cieco, Unam, Gira, Mundiprensa, Fundación Instituto de agricultura ecológica y sustentable, España, Valencia. pp. 200.

Bacon, C.M.; Getz, C.; Kraus, S.; Montenegro, M.; Holland, K. 2012. The Social Dimensions of Sustainability and Change in Diversified Farming Systems. Ecology and Society, 17 (4): 41.

Bacon C.M.; Sundstrom, W.A.; Flores G.M.E.; Méndez, V.E.;

Santos, R.; Goldoftas, B.; Dougherty I.

2014. Explaining the 'hungry farmer paradox': Smallholders and fair trade cooperatives navigate seasonality and change in Nicaragua's corn and coffee markets. Global Environmental Change, 25:133-149.

Borkhataria, R.; Collazo, J.A.; Groom, M.J.; Jordan-García A. 2012. Shade-grown coffee in Puerto Rico: Opportunities to preserve biodiversity while reinvigorating a struggling agricultural commodity. Agriculture, Ecosystems and Environment, 149: 164-170.

Castro-Tanzi, S.; Dietsch, T.; Urena, N.; Vindas, L.; Chandler, M. 2012. Analysis of management and site factors to improve the sustainability of smallholder coffee production in Tarrazú, Costa Rica. Agriculture, Ecosystems and Environment, 155: $172-181$.
Cerdán, C.R.; Rebolledo, M.C.; Soto, G.; Rapidel, B.; Sinclairet, F.L. 2012. Local knowledge of impacts of tree cover on ecosystem services in smallholder coffee production systems. Agricultural Systems, 110: 119-130.

Damiani, O.

2005. Adversidad y cambio: estrategias exitosas de pequeños productores de café en Centroamérica. Ed. 1, San José, Costa Rica. ISBN: 9968-866-46-6, pp. 48.

Danse, M. y Velleman, S.

2007. Small-scale Farmer Access to International Agri-food Chains. Greener Management International, 2005 (51): 39-52.

Donovan, J. y Poole, N.

2014. Changing asset endowments and smallholder participation in higher value markets: Evidence from certified coffee producers in Nicaragua. Food Policy, 44: 1-13.

Elder, S.D.; Zerriffi, H.; Billonet, Le Philippe.

2013. Is Fairtrade certification greening agricultural practices? An analysis of Fairtrade environmental standards in Rwanda. Journal of Rural Studies, 32: 264-274.

Gallopín, G.

2003. Sostenibilidad y desarrollo sostenible: un enfoque sistémico. Serie Medioambiente y Desarrollo 64. CEPAL. Santiago de Chile, pp. 44.

Gillison A.N.; Liswanti, N.; Budidarsono, S.; Noordwijk van

M.; Tomich, T.P.

2004. Impact of Cropping Methods on Biodiversity in Coffee Agroecosystems in Sumatra, Indonesia. Ecology and Society, 9 (2): 7.

Hart, R.D.

1985. Conceptos básicos sobre agroecosistemas. CATIE, Turrialba, Costa Rica, pp. 160.

Hauserman, $\mathrm{H}$.

2014. Maintaining the Coffee Canopy: Understanding Change and Continuity in Central Veracruz. Hum. Ecol., 42: 381-394.

Holt-Giménez, E.

2008. Campesino a Campesino, Voces de Latinoamérica Movimiento Campesino para la Agricultura Sustentable. SIMAS, Managua, Nicaragua, pp. 312.

Fischersworring H.B. y Robkamp, R.R.

2001. Guía para la Caficultura Ecológica. Tercera edición, Editorial López. BMZ de la República Federal de Alemania, pp. 153. 
Katlyn S.M.; Méndez, V.E.; Olson, M.B.

2013. 'Los meses flacos': seasonal food insecurity in a Salvadoran organic coffee cooperative. The Journal of Peasant Studies, 40 (2): 457-480.

Masera O., Astier, M.; López-Ridaura, S.

1999. Sustentabilidad y manejo de recursos naturales: el marco de evaluación MESMIS. Mundiprensa, GIRA, UNAM, México D.F.: 160 pp.

Mendén V.E.; Bacon, C.M.; Olson, M.; Petchers, S.; Herrador,

D.; Carranza, C.; Trujillo, L.; Guadarrama-Zugasti, C.; Cordón,

A.; Mendoza. A.

2010. Effects of Fair Trade and organic certifications on small-scale coffee farmer households in Central America and Mexico. Renewable Agriculture and Food Systems, 25 (3): 236-251

Meylan, L.; Merot, A.; Gary, C.; Rapidel, B.

2013. Combining a typology and a conceptual model of cropping system to explore the diversity of relationships between ecosystem services: The case of erosion control in coffee-based agroforestry systems in Costa Rica. Agricultural Systems, 118: 52-64.

Moguel, P. y Toledo, V.M.

1999. Review: Biodiversity Conservation in Traditional Coffee Systems of Mexico. Conservation Biology, 13 (1): 11-21.

Nonato de Souza, H.; Goede, de Ron G.M.; Brussaard, L.;

Cardoso, I.M.; Duarte, E.M.G.; Fernandes, R.B.A.; Gomes,

L.C.; Mirjam M. Pulleman, M.M.

2012. Protective shade, tree diversity and soil properties in coffee agroforestry systems in the Atlantic Rainforest biome. Agriculture, Ecosystems and Environment, 146: 179-196.

Perfecto, I.; Vandermeer, J.H.; Bautista, G.L.; Nunez, G.I.;

Greenberg, R.; Bichier, P.; Langridge, S.

2004. Greater predation in shaded coffee farms: the role of resident neotropical birds. Ecology, 85 (10): 2677-2681.
Pretty, J.

2008. Agricultural sustainability: concepts, principles and evidence. Philosophical Transactions, The Royal Society, 363: 447-465.

Rahn, E. ; Läderach, P.; Baca, M.; Cressy, C.; Schroth, G.; Malin,

D.; Rikxoort van , H.; Shriver, J.

2013. Climate change adaptation, mitigation and livelihood benefits in coffee production: where are the synergies? Mitig Adapt Strateg Glob Change, 19: 1119-1137.

Ruben, R. y Fort, R.

2012. The Impact of Fair Trade Certification for Coffee Farmers in Peru. World Development, 40 (3): 570-582.

Sarandón, S.J.

2002. El desarrollo y uso de indicadores para evaluar la sustentabilidad de los agroecosistemas. En: Agroecología: El camino hacia una agricultura sustentable. Ediciones Científicas Americanas. Argentina: 350-394.

Sarandón, S.J.; Flores, C.C.

2009. Evaluación de la sustentabilidad en agroecosistemas: una propuesta metodológica. Agroecología, 4: 19-28.

Soini E.

2005. Changing livelihoods on the slopes of Mt. Kilimanjaro, Tanzania: Challenges and opportunities in the Chagga homegarden system. Agroforestry Systems, 64: 157-167.

Speelman E.N.; Groot J.C.J.; García-Barrios, L.E.; Kok, K.; Keulen van H.; Tittonell, P.

2014. From coping to adaptation to economic and institutional change-Trajectories of change in land-use management and socialorganization in a Biosphere Reserve community, Mexico. Land Use Policy 41: 31-44.

Urrútia G, Bonfill X.

2010. Declaración PRISMA: Una propuesta para mejorar la publicación de revisiones sistemáticas y metaanálisis. MedClin (Barc), 135 (11): 507-511. 
